\title{
ICRF system efficiency
}

\author{
$\underline{\text { H. Faugel }}^{1}$, V. Bobkov ${ }^{1}$, H. Fünfgelder ${ }^{1}$, J. M. Noterdaeme ${ }^{1,2}$, A. Messiaen $^{3}$, D. Van Eester ${ }^{3}$, \\ ASDEX Upgrade Team ${ }^{1}$ and EUROFUSION WPHCD Team and MST1 Teams \\ 1 Max-Planck-Institut für Plasmaphysik, EURATOM Association, Garching, Germany \\ 2 Applied Physics Department, Ghent Univerity, Belgium \\ 3LPP-ERM/KMS, Brussels, Belgium
}

\begin{abstract}
The efficiency of heating and current drive systems is one of the key parameters for a successful operation of fusion demonstration power plants like DEMO. In an earlier review article, overall plug efficiencies of H \& CD systems were estimated at $20-30 \%,[1]$. We present here a detailed breakdown based where possible on experimental data for the overall efficiency (plug to power in plasma) of ICRF (ion cyclotron range of frequencies) systems: 1) the technical efficiencies (RF generator, transmission lines, losses in antenna); 2) the interface efficiency (hardware/ plasma) and 3) heating efficiency (absorption in plasma). This leads currently to an overall efficiency for heating in the range $40 \%$ to $55 \%$. Future improvements can lead to an overall efficiency of up to 69 $\%$. In a second step we address the current drive efficiency (in terms of kA/MW absorbed).
\end{abstract}

Keywords: heating, current drive, ICRF, ICRH, efficiency

\section{Introduction}

The efficiency of the heating and current drive systems is a key parameter for the successful operation of a fusion power plant. In an often cited paper about research and development issues for DEMO an overall plug efficiency for ICRF of $20-30 \%$ was mentioned [1]. We present data of currently archived efficiencies from plug to power in the plasma of the ICRF system of the ASDEX Upgrade (AUG) tokamak and show that further developments can lead to future improvements.

\section{Efficiency from plug to power in plasma}

Our overall efficiency is defined as the ratio of the power in the (central) plasma to the electrical input power. We have split this up in 3 components: 1) the technical efficiencies 2) the interface efficiency 3) the heating efficiency. For each component the achieved and the achievable numbers are discussed, whereby the numbers in bold are summarized in table 3 .

\subsection{Technical efficiency}

This number itself includes the efficiencies of producing the RF energy (power supplies and RF generator), the efficiency of the transport to the antenna (thus taking into account the losses in the transmission lines and the antennas).

\subsubsection{Power supplies}

The efficiency of today's power supplies is high when they are used close to their maximum ratings. High voltage power supplies based on thyristors reach efficiencies up to $\mathbf{9 5 \%}$ [2]. Newer power supply topologies like the pulse step modulator (PSM) reach up to $\mathbf{9 7 \%}$ [3]. The PSM power supplies can benefit from new wide band gap semiconductors that allow a significant reduction of the number of modulator stages needed.

\subsubsection{RF generation efficiency}

Amplifiers that are currently used by ICRF systems consist of one or two semiconductor stages with power levels of $100 \mathrm{~W}$ up to several $\mathrm{kW}$ followed by two or three vacuum tubes stages (triodes or tetrodes) reaching an output power of up to $2 \mathrm{MW}[4,5,6]$. These amplifiers are operated in the so called Class-B mode, allowing a maximum efficiency of the amplifier of $78.5 \%$ [7]. The operation of the amplifier needs subsystems like power supplies for the filaments and for the grids of the electron tubes, pumps and ventilators for cooling etc.. To calculate the technical efficiency for the ASDEX Upgrade ICRF system we included the generator efficiency, the ICRF subsystems such as the air supply for the transmission lines, the vacuum system for part of the feeding lines that are under vacuum and the control and data acquisition. These consume about $56 \mathrm{~kW}$ per RF generator for the $2 \mathrm{MW}$ generators of AUG. The efficiency of the AUG RF generator \#1 operated at $30 \mathrm{MHz}$ with an output power of up to $1 \mathrm{MW}$ during a $2 \mathrm{~T} \mathrm{H}$-mode discharge of ASDEX Upgrade is shown in Fig.1. During the second half of the discharge the plasma-antenna distance was changed several times. Although a 3-dB hybrid was used to decouple the changes of the antenna impedance from the RF generator, the small residual load changes at the generator cause a variation of the RF generator efficiency, between $65 \%$ and $\mathbf{7 5 \%}$, which is about the maximum achievable with Class-B amplifiers (78.5\%). To operate the RF generator with maximum efficiency an adapted anode voltage as discussed in [8] is vital. Higher efficiencies of up to $80 \%$ can be reached by operating this kind of amplifier in Class-C mode [9]. It requires a redesign of internal power supplies and the driver stage. The lifetime of the tetrodes is however likely to decrease in this operation mode because of the higher power dissipation at the grids. Today's semiconductor RF power amplifiers are reaching an output power of tens of $\mathrm{kW}$ and are widely used for broadcast applications [10] as they are more reliable and show much lower maintenance costs, even though the efficiency in some broadcast application does not exceed those of tube amplifiers. In recent years a few high power amplifiers have been designed for particle accelerators, one of the earliest reaching up to $190 \mathrm{~kW}$ at $352 \mathrm{MHz}$, used at the SOLEIL synchrotron [11]. 
Supervision circuits allow easy failure detection and the modular design allows short repair times.

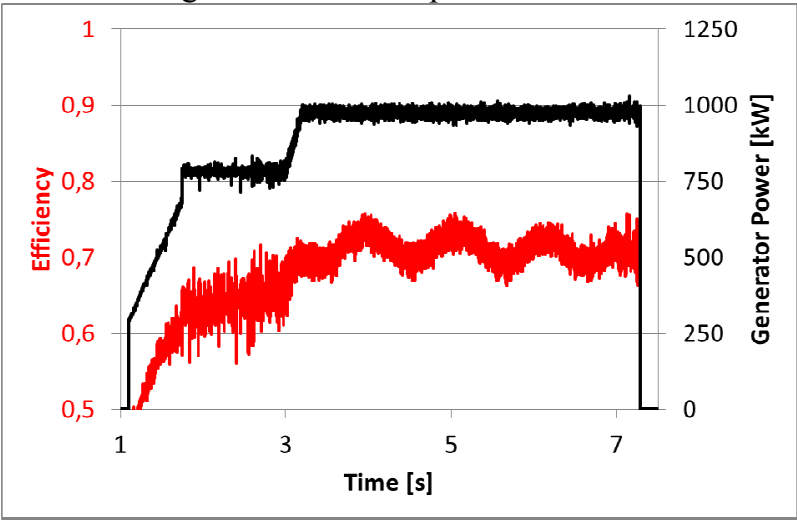

Fig.1 The efficiency (red) and output power (black) of the ICRF generator \#1 during shot 32141, inclusive all subsystems.

The further development of LDMOS (laterally diffused metal oxide semiconductor) devices allow a reduction of the number of LDMOS transistors from 724 to 256, as the output power of each transistor was increased from 350 to $650 \mathrm{~W}[11,12]$ providing a total $\mathrm{rf}$ power of 190 $\mathrm{kW}$ respectively $150 \mathrm{~kW}$. New design approaches to combine the power of individual amplifiers lead to lower losses and more compact design [13]. Currently $2 \times 2$ MW $200 \mathrm{MHz}$ rf amplifiers using in total 5120 LDMOSFETs are facing its completion [14]. New wide band gap semi- conductors like GaN and SiC HEMT (HighElectron-Mobility Transistor) with characteristics that outperform the currently used Si LDMOS, would even allow moving from the linear class B amplifier topology with a maximum theoretical efficiency of $78.5 \%$ to the switch mode class $\mathrm{E}$ amplifier topology which can reach an efficiency of $88 \%$ [15]. To take into account the power needed for auxiliaries, we multiply this $88 \%$ with the ratio of achieved efficiency on ASDEX Upgrade (75\%) to the maximum achievable $(78.5 \%)$, giving $84 \%$. 1.1.3. Matched transmission line and waveguides ICRF systems typically use coaxial transmission lines (TL) with characteristic impedances between $25 \Omega$ and $50 \Omega$ to transfer power to the antenna. A minimum of attenuation can be achieved using a coaxial TL with 77 $\Omega$. While the losses of the inner conductor are about the same as on a $50 \Omega$ transmission line, the smaller diameter of the inner conductor leads to a much higher temperature, therefore $50 \Omega$ transmission lines are used as a good compromise. Fig. 2 shows the attenuation and steady state power handling limits for $50 \Omega$ coaxial transmission lines with $\mathrm{Cu}$ inner and $\mathrm{Al}$ outer conductor for $120{ }^{\circ} \mathrm{C}$ inner conductor temperature and $40^{\circ} \mathrm{C}$ ambient temperature. The losses on the inner conductor and the weak thermal conductivity of air are the limiting factor for continuous operation. Successful tests with actively cooled conductors (see table 2,12 " cooled) were made [16]. A power of $6 \mathrm{MW}$ was transmitted at 49.6 $\mathrm{MHz}$. At the DEMO frequency $(74 \mathrm{MHz})$ this would correspond to a capability of transmitting $4.25 \mathrm{MW}$ (lower power, to keep the losses the same at the higher frequency). Accessing the inner conductor for cooling remains complicated. Table 2 shows an overview of different TL sizes and the minimum number of TLs that would be needed for a $50 \mathrm{MW}$ ICRF system for DEMO.

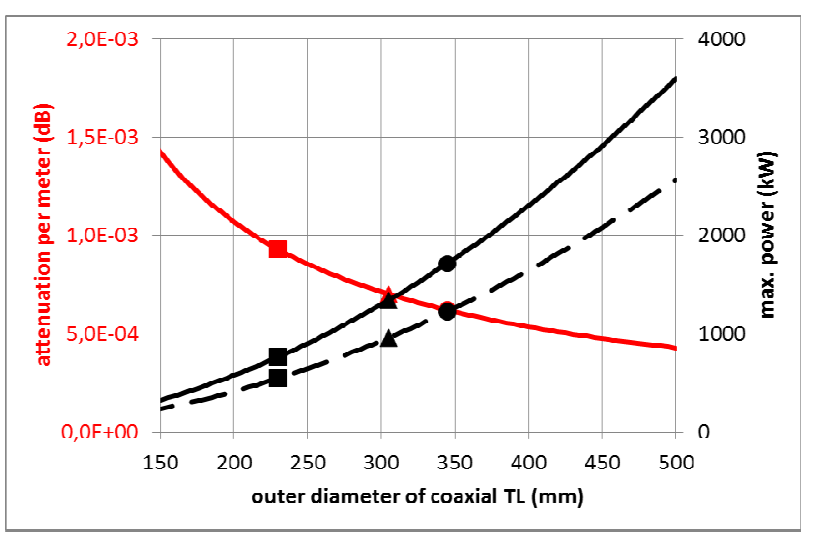

Fig.2 Attenuation (red) and steady state power handling capability of a matched $50 \Omega$ coaxial transmission line with $\mathrm{Cu}$ inner and $\mathrm{Al}$ outer conductor at $74 \mathrm{MHz}$ as function of diameter of the outer conductor. The top curve is the maximum allowable power for $120^{\circ} \mathrm{C}$ at the inner conductor and $40{ }^{\circ} \mathrm{C}$ ambient temperature of a coaxial line with 3 bar air pressure, the bottom curve is valid for an unpressurized TL. The symbols correspond to the TL types of Table 2 (see outer diameter).

Table 2: power limits and losses for different TLs at $74 \mathrm{MHz}$

\begin{tabular}{|l|l|l|l|l|}
\hline TL type & $\begin{array}{l}\text { outer } \\
\text { diam. } \\
{[\mathrm{mm}]}\end{array}$ & $\begin{array}{l}\text { max. } \\
\text { power } \\
{[\mathrm{kW}]}\end{array}$ & $\begin{array}{l}\text { \# TLs } \\
\text { for 50 } \\
\text { MW }\end{array}$ & $\begin{array}{l}\text { losses/m @ } \\
50 \mathrm{MW} \\
\text { (with \# of } \\
\text { TL needed) }\end{array}$ \\
\hline RL100-230 & 230 & 719 & 70 & $12.5 \mathrm{~kW}$ \\
\hline $12 " \boldsymbol{\Delta}$ & 305 & 1264 & 40 & $9.39 \mathrm{~kW}$ \\
\hline RL 150-345 • & 345 & 1618 & 31 & $8.31 \mathrm{~kW}$ \\
\hline $12 "$ cooled & 305 & 4251 & 12 & $9.39 \mathrm{~kW}$ \\
\hline
\end{tabular}

The typical length of the matched TLs for an ICRF system is about $100 \mathrm{~m}$, and, with the values of Table 2 , the losses are between 1.6 and $2.5 \%$. In the unmatched section of the transmission line standing waves are present, which increases the losses depending the reflection factor $\Gamma$. The total losses can be reduced by a careful selection of the impedance of the transmission line with respect to the impedance of the antenna. A $\lambda / 4$ transformer connected close to the antenna can greatly help to minimize the losses in the unmatched section. High radiation levels of the ITER and DEMO can cause an increase of the dielectric losses of ceramic insulators used to center the inner conductors. This problem can be solved by using conducting $\lambda / 4$ long supports of the inner conductors; a relative bandwidth of $+/-5 \%$ of the center frequency can be easily achieved with supports that are spaced $n * \lambda / 4$ apart. This setup also allows access for gaseous or liquid coolant to the inner conductor. Efficiencies for the transmission lines are thus of the order of 97.5 to $98.4 \%$ at a VSWR of 1 , a VSRW of 1.5 would decrease the efficiency to $97.3 \%$ respectively $98.2 \%$.

The design of ICRF systems at ITER and DEMO are very demanding as it requires the transmission of several tens of megawatt to the antennas. While this can be done by using multiple coaxial TLs in parallel, and some optimization in terms of overall losses is possible with respect to the number and size of the TL's (see table 2), 
an alternative solution using a rectangular waveguide where the power is dissipated at easy accessible surfaces could be an option. This avoids the problem of having to cool the central conduction and even reduces the losses. The largest standard rectangular waveguide size available is WR2300, which allows operation between 320 and $490 \mathrm{MHz}$. As DEMO should operate between 66 and $74 \mathrm{MHz}$ [17], a larger non-standard waveguide measuring $3000 \mathrm{~mm}$ by $500 \mathrm{~mm}$ would result in a more compact design than number of needed parallel coaxial TLs. It is also cheaper and easier to install. The transmission losses of such a waveguide at $74 \mathrm{MHz}$ are one fourth compared to $50 \Omega$ TLs with a $345 \mathrm{~mm}$ outer conductor. Such a waveguide can be directly attached to an amplifier with a cavity combiner. Waveguide efficiencies could then be up to $\mathbf{9 9 \%}$.

\subsubsection{Antennas and (short) unmatched section}

Recent ICRF antennas use a relatively small number of antenna loops to couple RF-power to the plasma, with an average power density of about $2 \mathrm{MW} / \mathrm{m}^{2}$ and a coupling resistance of the order of a few Ohm per antenna loop. The losses of the antenna can be estimated by comparing the vacuum impedance of the antenna with the impedance during plasma operation, typical ratios are around 0.1 which means that $10 \%$ of the power is lost in the antenna (eff. 90\%). For DEMO, one a approach was using a $360^{\circ}$ distributed antenna fully integrated in the blanket [18, 19 and 20]. The local power density of a distributed antenna is lower than for current designs and the antenna can be designed for small losses. Since the coupling resistance of the antenna array is close to the characteristic impedance of the transmission line, the power losses and the maximum voltage in the unmatched antenna feeding lines are reduced due to a low VSWR. The low VSWR demands only a simple matching system like a $\lambda / 4$ transformer or no matching system at all. Currently a conventional plug-in ICRF antenna is foreseen for DEMO, therefor we take an antenna efficiency of $\mathbf{9 0 \%}$.

\subsection{Interface efficiency}

We define interface efficiency as the power that reaches the main plasma divided by the power that leaves the antenna. This thus takes into account power losses in the scrape-off layer. In present-day machines, good absorption in the (confined) plasma of the power leaving the antenna in a single transit is not always guaranteed. However, since the tokamak vessel acts as a Faraday cage from which RF power cannot escape, all power launched from the antennas needs to be absorbed. Crudely speaking the power density $\mathrm{P}_{\mathrm{d}}$ absorbed locally in a plasma is the product of the electric field $\mathbf{E}$ amplitude squared and a plasma dependent factor $\mathrm{C}_{\mathrm{p}}$ involving density, temperature, etc.: $\mathrm{P}_{\mathrm{d}}=\mathrm{C}_{\mathrm{p}}|\mathbf{E}|^{2}$. When the plasma absorptivity is low, $\mathrm{C}_{\mathrm{p}}$ is small and the electric fields need to be large to ensure that the above equality is satisfied for a given amount of power launched. Since the fast wave is evanescent in the edge and hence the electric field is exponentially decaying between the launcher and the plasma, RF coupling very sensitively depends on the density profile. In large size machines, the distance between the last closed flux surface and the launcher is prohibitive and gas injection modules are required to ensure the distance between the antenna and the fast wave cutoff is an engineering parameter rather than being one imposed by spontaneous processes. In the low density edge, the $|\mathbf{E}|$ is largest close to the launchers and the risk for parasitic absorption is highest there. Optimizing the core plasma heating scheme (increasing $\mathrm{C}_{\mathrm{p}}$ ) is thus the best way to limit wave induced plasma wall interaction. How good the core (being here defined as the region where the plasma is confined) plasma heating scheme is, can be quantified using the single transit absorption concept. The single transit absorption is a calculated value: the ratio of power absorbed in two passes, from the antenna through the plasma, and back from the inner side of the plasma on the high field side to the antenna (we use double pass since absorption in a single pass can be asymmetric due to cut-off and mode conversion layers). This calculated value of the "single transit" absorption takes into account absorption in the confined plasma on ions and electrons. One can make an estimate of the losses in the edge, by assuming that a fraction of the power, not absorbed in each pass is lost in the outer edge. For AUG we find experimentally, that scenarios with calculated single transit absorption of $15 \%$, do not lead to operational problems and that $>$ $\mathbf{7 0 \%}$ of the power is absorbed in the plasma [25]. The interface efficiency is then $70 \%$ and the total loss in the edge thus $<30 \%$. With single transit absorption of $15 \%$, we can calculate a loss per double pass in the (outer) edge of $6.5 \%$ (this leads asymptotically to a cumulative loss in the edge of $29 \%$ ). In large size machines, the heating efficiency is commonly good unless evanescence layers prevent the launched waves to reach the core plasma (see e.g. [21, 22]): In ITER, wave absorption during a single transit of the RF wave over the plasma will be in excess of $80 \%$ [27] with the appropriate choice of frequency. Taking the same values of loss per pass $(6.5 \%)$ also in larger machines (which is likely an overestimate, because of the smaller extend of the scrape-off layer to the main plasma), and using a single transit absorption value of typically $80 \%$ [27], we find for the total losses in the edge less than $5 \%$ (because fewer passes are needed to absorb all the power). The corresponding interface efficiency is thus $\mathbf{9 5 \%}$. Strictly speaking, the efficiency cannot be computed in this way: although the tokamak acts as a (metal) Faraday cage forcing the power launched from the antenna to be absorbed inside the vessel, parasitic absorption in the edge is more important when the core absorption is weak than when it is strong. Waves re-incident on the antenna modify the current pattern on the antenna as well as the phase and amplitude of the vacuum waves in between the generator and the antenna. Hence the fate of the power in one subregion influences the behavior in other subregions. Nevertheless, the attempt to isolate the various subregions allows us to get a first estimate of the role played by the different regions while clearly leading to an underestimate of the efficiency in the large machines.

\subsection{Heating efficiency}

The heating efficiency is defined as ratio of the power 
that is useful to heat the plasma to the power that reaches the confined plasma (note that losses in the edge is taken into account in the interface efficiency). For ICRF, this efficiency takes for example into account that, for certain scenarios, one could heat in the confinement region ions to very high energy that are lost before they could transfer their energy to bulk ions and electrons. For present day machines, because of the scenario used (minority heating) and the relative small value of the confining plasma current and machine size, this can indeed be a concern, though a minor one. In machines of the size of ITER and DEMO, where a large fraction of the heating is done through fusion-born $\alpha$ particles, the machines are designed to confine the very energetic $\alpha$ particles. The RF heating scheme chosen will avoid unconfined ions and be resistant against non-desired wave-induced further acceleration of the $\alpha$ 's: second harmonic $\mathrm{T}$ heating, possibly with ${ }^{3} \mathrm{He}$ minority. Since the machines are designed to confine the very energetic $\alpha$ 's, losses related to the heating of non-confined energetic particles is thus not an issue [28]. The heating efficiency in those cases can confidently be set to $\mathbf{1 0 0 \%}$.

\section{Current drive efficiencies}

After the ramp-up phase of the discharge, the RF heating system could then be exploited for another purpose: current drive, as modeling shows that an ignited plasma can be solely heated by the slowing down of the $\alpha$ population [28]. The physics current drive efficiency is a number that calculates the driven current per power absorbed in the confined plasma. To get the relevant number for the power balance of a reactor (overall current drive efficiency) one also needs to take into account the efficiencies from the plug to the power in the plasma. To first order, one can take for this later the value obtained in the heating section. The local current drive efficiency $J / P_{d}$ of an RF heating scheme is usually estimated relying on an approximate expression proposed by Ehst [23]. Here $J$ is the current density driven by a power density $\mathrm{P}_{\mathrm{d}}$ passed on from the waves to the electrons. Ehst's expression accounts for the fact that trapped particles do not contribute to the current, at least not in the zero orbit width limit. As a consequence, driving a current close to the plasma core is more efficient than driving a current off-axis, and driving a current on the high field side is more efficient than driving it on the low field side. Integrating over the obtained current density profile provides the total current that can be driven for a given input power. Good electron absorption is a prerequisite for driving current. Ideally, $w=\omega /\left(\mathrm{k}_{/ /} \mathrm{v}_{\text {th,e }}\right)$ is close to but slightly higher than 1 (at 1 , the electron damping is optimal). Accounting for that condition and for profiles deemed realistic for DEMO, current drive efficiencies of the order of 3040kA per MW absorbed on the electrons have been calculated for equatorial launch and up to $60 \mathrm{kA} / \mathrm{MW}$ for near-top launch [24]. However, only if the antenna spectrum $\left(\mathrm{k}_{/ /}\right)$can be adapted when the plasma temperature $\left(\mathrm{v}_{\mathrm{th}, \mathrm{e}}\right)$ varies one can maintain optimal efficiency during a discharge.

\section{Summary and Conclusions}

Overall plug to power in the plasma efficiencies are already rather high in present experiments such as ASDEX Upgrade. Further improvements are to be expected in DEMO (table 3), in particular due to its larger size and better absorption. It should be also noted that the overall technical efficiency of an ICRF system cannot be archived at the maximum output power as amplifiers tend to decrease their efficiency under these conditions. Physics current drive efficiencies are low but for the overall current drive efficiency this is somewhat compensated by the high plug to power efficiency. Similarly, low overall current drive efficiency numbers are obtained for other heating systems like electron cyclotron systems (see e.g. [25]), indicating that novel ideas are required for at economically viable steady state tokamaks.

Table 3: reached and expected total ICRF heating efficiency

\begin{tabular}{|l|l|c|c|}
\hline Chap. & & $\begin{array}{l}\text { efficiency } \\
\text { at AUG }\end{array}$ & $\begin{array}{l}\text { for } \\
\text { DEMO }\end{array}$ \\
\hline 1.1 .1 & power supply & 0.95 & 0.97 \\
\hline 1.1 .2 & RF generator & 0.75 & 0.84 \\
\hline 1.13 & transmission line & 0.98 & 0.99 \\
\hline 1.1 .4 & Antenna & 0.90 & 0.90 \\
\hline & total technical efficiency & 0.63 & 0.73 \\
\hline 1.2 & interface efficiency & 0.70 & 0.95 \\
\hline 1.3 & plasma heating efficiency & 1 & 1 \\
\hline & $\begin{array}{c}\text { total heating efficiency } \\
\text { (power in central plasma/plug power) }\end{array}$ & $\mathbf{0 . 4 4}$ & $\mathbf{0 . 6 9}$ \\
\hline 2 & $\begin{array}{c}\text { phys. current drive efficiency } \\
\text { (current/absorbed power) in A/W }\end{array}$ & $\mathrm{n} / \mathrm{a}$ & 0.060 \\
& $\begin{array}{c}\text { total current drive efficiency } \\
\text { (current / plug power) in A/W }\end{array}$ & $\mathrm{n} / \mathrm{a}$ & $\mathbf{0 . 0 4 1}$ \\
\hline
\end{tabular}

\section{Acknowledgments}

This work has been carried out within the framework of the EUROfusion Consortium and has received funding from the Euratom research and training programme 2014-2018 under grant agreement No 633053. The views and opinions expressed herein do not necessarily reflect those of the European Commission.

\section{References}

[1] J. Pamela et al, DOI: 10.1016/j.fusengdes.2009.02.028

[2] J. Bradley et al, Operational experience with two types of 2MW HVDC power supplies on LEDA, Proceedings of the 1999 IEEE Particle Accelerator Conference, p.1010

[3] P. Patel et al, DOI: 10.1109/TPS.2012.2227330

[4] W. Schminke et al, Upgrading of ICRH generators for ASDEX/W VII and ASDEX Upgrade, 12th SOFE, 1987

[5] H. Fünfgelder et al, DOI: 10.1063/1.3664943

[6] F. Pompon et al, DOI: 10.1063/1.3664942

[7] A. Grebennikov, N. O. Sokal, Switchmode RF Power Amplifiers, ISBN : 978-0-7506-7962-6, p. 7 - 15

[8] Ch. Imboden et al, 2x2 MW ICRH amplifier system, 18th SOFT (1994), p. 553 - 556

[9] W. Schmincke, The Development of Plasma-Heating Equipment and Classical High-Power Broadcast Transmitter Technology, 15th SOFT (1988)

[10] https://indico.cern.ch/event/472685/contributions/2196879

[11] P. Marchand et al,DOI:10.1103/PhysRevSTAB.10.112001

[12] J. Jacob, DOI: 10.5170/CERN-2015-003.197

[13] https://indico.cern.ch/event/472685/contributions/2196878

[14] https://indico.cern.ch/event/472685/contributions/2196909

[15] A. Grebennikov, N. O. Sokal, Switchmode RF Power Amplifiers, ISBN : 978-0-7506-7962-6, p. 218 - 227

[16] R. H. Goulding et al, DOI : 10.1063/1.4864565

[17] D. Gallart et al, DOI : 10.1063/1.4936502

[18] A. Garcia et al, DOI : 10.1063/1.4936509 
[19] G. Bosia, DOI : 10.1016/j.fusengdes.2014.12.008

[20] A. Messiaen, R. Ragona, Study of the coupling of distributed ICRH antenna and of its optimization for heating of large machines as DEMO, EPS (2016)

[21] D. Van Eester et al., Nucl. Fusion 42 (2002) 310-3286

[22] W. Zhang et al, DOI : 10.1088/1741-4326/aaddae

[23] D.A. Ehst and C. Karney, Nuclear Fusion 31 (1991) 1933

[24] Ye. O. Kazakov et al., Plasma Phys. Control. Fusion 57 (2015) 025014

[25] Zohm H, et al. Proc. 40th EPS Conf. on Plasma Physics, ECA 37D, O3.108,

http://ocs.ciemat.es/EPS2013PAP/pdf/O3.108.pdf

[26] J.-M. Noterdaeme et al., RF Power in Plasmas, AIP Conference Proceedings 289 (1993) 12-22

[27] D. Van Eester et al., Nucl. Fusion 42 (2002) 310-328

[28] D. Van Eester et al., Nucl. Fusion 59106051 